\title{
MULTI-LEVEL HIERARCHICAL SYSTEM TO TAKE INTO ACCOUNT UNCERTAINTY IN MULTI-CRITERIA DECISION MAKING PROCESS
}

\author{
Vladimir I. Kalika \\ Natural Resources and Environmental Research Center, Haifa University, \\ Mount Carmel, 31905 Haifa-Israel, kalika@econ.haifa.ac.il
}

Keywords: multi-criteria decision making, uncertainty, multi-level hierarchical system, scenarios, Monte Carlo simulation.

Summary: A new approach is proposed to select a predetermined number of "reasonable" alternatives from their vast initial set according to an arbitrary (but finite) number of optimization criteria and accounting for uncertainty factors. This approach uses an intuitive methodology developed to account for uncertainty. The methodology is based on performing multi-variant computations (MVC) and finding their "stable-optimal" solutions. All this is performed in a framework of a hierarchical multi-level system of MVC series, where each level of this system includes a set of appropriate scenarios, having the same nature.

\section{Introduction}

The considered problem of multi-criteria decision making (MCDM) could be characterized as follows: There are a considerable (often, a vast) number of initial alternatives, that allows, in principle, to form a full initial set of alternatives (this set is named as the ISA). The purpose of this considered MCDM process is to select a predetermined number of "reasonable" (the best in a certain sense) alternatives from the ISA, where these "reasonable" alternatives set (named as the RAS) could be used to find a final solution of the problem (in the form desired for decision makers) on a basis of the RAS analysis using additional qualitative and quantitative criteria and estimates. The required set RAS is to be found with joint accounting for all (a priori given) objectives, expressed by multiple criteria, whose number maybe, in principle, unlimited (but finite). To account for all these criteria as applied to each possible initial alternative from the discrete ISA, the appropriate criteria calculation models could be developed. Using these models, a criteria assessment vector can be determined for each alternative from the ISA, where this vector is represented by one numerical value for the considered pair "alternative-criterion". Thus, each criteria assessment vector corresponds to one initial alternative and conversely, and, then, an initial set of such vectors (this set is named as the ISCAV) is interrelated with the ISA.

We will illustrate the considered type of problems on the sample of the problem of medium/long term power generation expansion planning for the conditions prevailing in Israel (Kalika and Frant, 1996, 1999, 2000). Here, each initial alternative represents a possible dynamic strategy of power generation expansion during the considered planning period (up to thirty years), and the "reasonable" alternative - a proper such strategy, selected on a basis of five optimization criteria (economic, reliability, three environmental), considered jointly. Since all possible strategies are mutually exclusive, their set is considered as an initial set of alternatives (ISA) and a set of the required proper strategies - as a "reasonable" alternatives set (RAS). In accordance with the problem conditions, the ISA includes a vast number of initial alternatives, and, thus, selecting a small (usually) RAS from a vast ISA according to the assigned multiple criteria is the purpose of this problem solution.

Various uncertainty factors are usually inherent in the problems of the considered type, as well as in the methods which could be developed to solve such problems. So, in the above mentioned illustrative problem uncertainty factors are due to various reasons, reflecting uncertainty inherent in: (a) the 
medium/long term planning itself, owing, first of all, to the long duration of the planning period; (b) forming the vast initial sets of alternatives (ISA) and criteria assessment vectors (ISCAV), where there are situations characterized by the difficulty or even the impossibility to present precise values of some initial parameters (e.g., many parameters are not reliable due to their nature); (c) constructing the solution methodology, that could involve necessity to overcome some peculiarities (e.g., choosing the proper optimization criteria and their weight values).

A solution process for each problem of the considered type should include the following basic stages: (1) constructing the ISA; (2) creating the ISCAV, interrelated with the ISA; (3) multi-criteria optimization on the space of ISCAV (ISA) to reach the required RAS using a developed intuitive multi-step man-machine procedure (MSMMP), which takes into account uncertainty factors.

The MSMMP methodology, developed by us (Kalika and Frant, 1996, 1999, 2000), reflects a quite universal procedure to be used for solving various problems of multi-criteria selection of a set of proper ("reasonable") alternatives from their discrete (finite) initial set (see section 2). Although many works consider similar problems (see, e.g., Koksalan, Karvan, Zionts, 1988; Rajabi, Hipel, Kilgour, 2000), our solution approach is a specific one, oriented on accounting jointly for all peculiarities (especially, a vast number of initial alternatives and uncertainty factors). This approach is based on: (a) performance of various multi-variant computation (MVC) series, based on varying calculation methods and procedures, used in MSMMP, as well as varying conditions and parameter values involved in the considered problem; (b) finding the "stable-optimal" solutions for all performed MVC series, realizing a multi-level hierarchical system of scenarios. Those scenarios, which reflect various calculation methods, consider the use of various versions of such multi-criteria optimization technique as TOPSIS (Massam, 1988), to be used alone or in combination with Pareto optimization (Steuer, 1986). All these versions represent our modifications of these (TOPSIS and Pareto optimization) techniques in order to account for uncertainty factors as a part of the developed general methodology to account for uncertainty (section $\mathbf{3}$ ).

The last version of MSMMP, presented in this paper (sections 3-4) and oriented, mainly, on using the criteria scalarization technique (TOPSIS), is interpreted as a six-level hierarchical system of scenarios (MVC series). It requires determination of the criteria weight values using decision-makers' estimates. Since they may be either subjective and not quite reliable or inappropriate at all (if the situations are characterized by a high level of uncertainty), this MSMMP solution methodology is based on: (a) considering the criteria weights as random variables, (b) finding the intervals of their possible values, (c) using a Monte Carlo simulation to determine the possible values of the random criteria weights. This MSMMP version as well as the whole above mentioned approach for solving the considered problems including the developed methodology of accounting for uncertainty are suitable for application to various problems in different fields of economics, environment, others (section 5).

\section{Mathematical Model of the Problem and its Specific Features}

The problem is presented by the following common multi-criteria optimization (MCO) model (see, e.g., Steuer, 1986):

$$
\begin{gathered}
\min z_{j}\left(x_{g}\right), j=1, . ., \mathrm{J} \\
\text { s.t. } \quad x_{g} \in A
\end{gathered}
$$

where $j$ [or $g$ ] is a number of objective [or alternative]; $x_{g}$ is a vector of variables or, that the same, $g$-point corresponding to $g$-alternative; $z_{j}\left[z_{j}\left(x_{g}\right)\right]$ is $j$-objective function or, that the same, $j$-criterion [its value for $g$-alternative]; $A=\left\{x_{g}\right\}$ is the ISA or, that the same, a set of all $g$-points $x_{g}$.

The specific features of model (1)-(2) as applied to our problem are as follows:

1. The space (2) of feasible solutions (the ISA) is a vast discrete set of $g$-points.

2. An arbitrary (although a finite) number Jof the considered criteria allows to form the discrete set ISCAV, interrelated with the ISA, where each vector from ISCAV, designated as $Z\left(x_{g}\right)=\left\{z_{j}\left(x_{g}\right), j=1, . ., \mathrm{J}\right\}$, corresponds to a certain $g$-alternative $x_{g}$. Thus, the MCO process (1) is performed on the ISCAV space:

$$
Z=\left\{Z\left(x_{g}\right) / x_{g} \in A\right\}
$$


3. The purpose of the problem (1)-(2) solution consists in finding the "reasonable" alternatives set (RAS) represented as the following subset of set (3):

$$
Z^{\wedge}=\left\{Z\left(x_{g}^{\wedge}\right) / x_{g}^{\wedge} \in R\right\}
$$

where $x_{g} \wedge$ is a "reasonable" alternative, the subset $R \subset A$ is the RAS.

4. Uncertainty factors, inherent to the problem, are considered as components of conditions for the model (1)-(2) and of the solution process proposed.

\section{Principles of Accounting for Uncertainty}

The main principle to take into account uncertainty factors in the considered approach, representing the first (basic) grade (aspect) of accounting for uncertainty, consists in: (a) performance of various multivariant computation $(M V C)$ series, based on varying calculation methods and procedures, used to solve the considered problem, as well as on varying conditions and parameter values involved in this problem; (b) finding the "stable-optimal" solutions for all performed MVC series, reflecting various scenarios in a framework of their multi-level hierarchical system (MLHS). The hierarchy in MLHS provides the order of performance of MVC series (scenarios), corresponding to various $l$-levels $(l=1, \ldots, \mathrm{L})$ of MLHS, in a framework of a general calculation process to solve the considered problem. In some cases, this hierarchy handles situations when MVC series, corresponding to a higher-order level of MLHS, vary the bounding parameters for MVC series of lower MLHS level (see sections 3.3, 4.1). According to this hierarchy principle, all possible (in MLHS) $l$-scenarios, corresponding to all possible $l$-levels $(l=1, . ., \mathrm{L})$ of the MLHS, may be classified under the following types of these l-levels: (1) external, representing the upper levels of $l$-scenarios $(l=1,2,3)$; (2) intermediate and (3) interior, reflecting the next lower $l$-levels of $l$ scenarios $(l>3)$. The second grade of accounting for uncertainty is connected with variation of $l$ scenarios inside of each $l$-level of MLHS. Such variations are specific for each $l$-level in MLHS. So, all $l$ scenarios, corresponding to the external $l$-levels $(l=1,2,3)$, reflect: (a) 1 -scenarios $(l=1)$ - various versions of used multi-criteria optimization techniques; (b) 2- or 3-scenarios $(l=2$ or 3$)$ - various possible versions of the ISA or the ISCAV. All $l$-scenarios of the intermediate and interior $l$-levels $(l>3)$ are specified by the peculiarities of each considered problem. The third grade of accounting for uncertainty is connected with random or directed variation of some parameter values in the problem.

We will consider in details all of the mentioned above grades of accounting for uncertainty. In a framework of considering $l$-scenarios of external type $(l=1,2,3)$, the main emphasis is given to 1 scenarios $(l=1)$, reflecting the versions of used multi-criteria optimization techniques, modified to account for uncertainty, as well as such modification of TOPSIS method (sections 3.1, 3.2). The interior and intermediate types of $l$-scenarios $(l>3)$ will be considered on a sample of the special case of MLHS, including its six levels $(l=1-6)$ and reflecting random variation (using a Monte Carlo simulation) of criteria weights in the modified TOPSIS method (see further sections 3.1-3.3, 4.1)

\subsection{Forming $l$-Scenarios of External Type}

All posssible 1-scenarios $(l=1)$ are constructed by varying the versions of multi-criteria optimization technique TOPSIS (Massam, 1988) to be used alone or in combination with Pareto optimization (Steuer, 1986). All these versions represent our modifications of these (TOPSIS and Pareto optimization) techniques in order to account for uncertainty factors.

Such modification of the TOPSIS technique (section 3.2) considers the criteria weight values either as determined or random variables (Techniques TD or TR) in accordance with the following cases:

TD1 or TD2) Equal weights (for all criteria) or their different possible values, determined using the decision makers estimates, are considered to obtain "rough" estimates of expected solutions in the situation of "full" or "partial" uncertainty with respect to decision makers" opinions in regard to the criteria weight values. 
TR1.r or TR2.r). The intervals of possible criteria weight values are constructed around each value (a "central point"), determined in the case TD1 or TD2, using $r$-version of the algorithm, developed to construct these intervals.

The Pareto optimization method, used in 1-scenarios, are modified to reflect a possibility of varying uncertainty ranges of domination bounds, which are used for comparison of each pair of criteria assessment vectors (alternatives).

Some possible combinations of the mentioned above cases allow to form the 1 -scenarios $(l=1)$ which may be expedient for use in MVC series of 1-level $(l=1)$ :

- $\quad 1 . D .1[1 . D .2])$ The performance of technique TD.1 [TD.2] only.

- $\left.\quad 1 *^{*} .1 . k\right)$ The successive performance of techniques TD.1 and TR.1 (TD.2 and TR.2). If a few $k$ versions $(k=1, \ldots, \mathrm{K})$ of the algorithm to construct the intervals of criteria weight values are considered, a set including $\mathrm{K}$ of the appropriate 1-scenarios $(l=1)$ can be formed.

- $\quad 1 . * 2 . k$ ). First, a Pareto optimization is performed to reduce a starting vast ISA to an intermediate $\mathrm{ISA}^{\wedge}$, having smaller size. Then any one of the scenarios $1 .^{*} .1 . k$ may be used to reduce the ISA ${ }^{\wedge}$ to the required final RAS.

Existence of various 2- or 3-scenarios ( $l=2$ or 3 ), representing versions of the ISA and ISCAV, is due to the impact of uncertainty factors inherent in the processes of the appropriate data accumulation and processing, etc. (e.g., variation in the ISCAV versions might reflect the doubt in choice of the proper criteria calculation models to be used in the calculation process).

\subsection{TOPSIS Method Modification Accounting for Uncertainty}

The main purpose of TOPSIS method (Massam 1988) is to select $g$-alternatives nearest to the " ideal solution" (IS). According to the model (1)-(3), this IS (WS - the "worst solution" is considered, too) presents the point $x^{\wedge}\left(x^{\wedge}\right.$ for WS) of the $g$-alternatives space where each $j$-coordinate $(j=1, . ., \mathrm{J})$ of this point is obtained by minimization (maximization) of the set $A=\left\{x_{g}\right\}$ on each $j$-criterion independently. Thus, IS (WS) corresponds to the point $Z^{\wedge}=\left\{z^{\wedge}, j=1, . ., J\right\}\left(Z^{\wedge}=\left\{z^{\wedge}{ }_{j}, j=1, . ., J\right\}\right)$ in the space (3), where:

$$
z^{\wedge}=\left\{\min z_{j}\left(x_{g}\right) / x_{g} \in A\right\} \quad\left(z^{\wedge}{ }_{j},=\left\{\max z_{j}\left(x_{g}\right) / x_{g} \in A\right\}\right), j=1, \ldots, \mathrm{J}
$$

Accounting for the relative "distances" between the IS and current $g$-alternative in the space (3), we determine for the $l$-scenario, corresponding to a TOPSIS version, a subset $S_{l}$ of "sub-ideal" or "suboptimal" alternatives, including $x_{g} \in A$ satisfying to the condition:

$$
\sum_{j=1}^{J}\left\{W_{j} *\left[z_{j}\left(x_{g}\right)-z^{\wedge}{ }_{j}\right] /\left(z^{\wedge} \wedge_{j}-z_{j}^{\wedge}\right)\right\}<d R
$$

where the assigned (a priori or by dialogue) limiting value $d R \quad(0<d R<1)$ reflects a relative estimate of "the proximity" of the considered g-alternative to IS.

To form all possible 1 -scenarios, corresponding to the above mentioned (section 3.1) versions of TOPSIS modifications, various cases of determination of the criteria weights $\left\{W_{j}, j=1, . ., \mathrm{J}\right\}$ for the formula (6) are considered, where the values $W_{j}$ are: (1) equal, i.e. $W_{j}=1 / \mathrm{J}, j=1, . . \mathrm{J}$ (technique TD.1); (2) given by decision makers either implicitly (technique TD.2) or as the criteria orders or priorities to be used for determining these $W_{j}$ values using the developed special heuristic algorithms; (3) considered as random variables $\left\{W_{j}^{\wedge}, j=1, . ., \mathrm{J}\right\}$ and if we want to obtain Q current values $\left\{W_{j}^{\wedge}[q], q=1, . ., \mathrm{Q}\right\}$ for each of these random variables, Q*J Monte Carlo simulations have to be performed (techniques TR.1 or TR.2).

\section{3 l-Scenarios of Intermediate and Interior Types for a Special Six-Level Hierarchical System of Multi-Variant Computations (MVC)}

According to the last MSMMP version, realized by a developed computer model (on "C"), the special six-level hierarchical system of MVC series is considered. This system includes six totalities of $l$ scenarios $(l=1, . ., 6)$, corresponding to their external $(l=1-3)$, intermediate $(l=4,5)$ and interior $(l=6)$ types. The totalities of external $l$-scenarios $(l=1-3)$, reflecting quite general positions, were considered early (sections 3.1, 3.2). Other $l$-scenarios $(l=4-6)$ should provide finding scalar sums of criteria, needed for 
TOPSIS, using random criteria weights and Monte Carlo simulations within the intervals of possible values for these weights. Here, the intermediate $l$-scenarios $(l=4,5)$ reflect various opportunities to construct the intervals of criteria weight possible values, where the interval "central points" are found in 4 -scenarios, the interval bounds - in 5-scenarios. Each interior 6-scenario $(l=6)$ reflects a possible criteria assessment vector, represented by a combination of the criteria weight values, obtained using $\mathrm{J}$ (the number of criteria) Monte Carlo simulations inside in the series of $\mathrm{J}$ intervals corresponding to a fixed pair of 4- and 5-scenarios. Thus, Q*J Monte Carlo simulations are performed for each pair of 4- and 5scenarios, if we want to consider Q such criteria assessment vectors or the same number (Q) of 6scenarios, corresponding to the considered pair of 4- and 5-scenarios.

\section{Calculation Process to Perform Multi-Level Hierarchical System of MVC series}

This process consists in reduction of the ISA to the RAS by means of multi-criteria optimization of the ISCAV (ISA) accounting for uncertainty factors. The process is interpreted as a multi-level hierarchical system (MLHS) of multi-variant computation (MVC) series, where each its l-level $(l=1, ., \mathrm{L})$ includes $\boldsymbol{a}$ totality of $\boldsymbol{l}$-scenarios, reflecting various operations of the same type corresponding to this $l$-level. A combination of $l$-scenarios, taken one at a time for each $l$-level $(l=1, . ., \mathrm{L})$, defines one full Scenario, corresponding to all considered $l$-levels $(l=1, \ldots, \mathrm{L})$. A totality of all full Scenarios, accepted in the calculation process, forms its MLHS as well as the calculation process as a whole. This calculation process, performed in the order of "bottom to top" or from the lowest L-level to the highest 1-level in accordance with each possible full Scenario, is based on finding a set of "stable-optimal" solutions (alternatives) for each $l^{\wedge}$-scenario, using a developed special procedure (see section 4.2). This procedure analyses the sets of "stable-optimal" ("sub-optimal" in some cases) subsets, obtained for $l$-scenarios of the lower $l$-levels $\left(l=l^{\wedge}+1, \ldots, \mathrm{L}\right)$, in order to find "stable-optimal" alternatives for $l^{\wedge}$-level, which should have the most frequencies of entering in these subsets. The resulting set RAS is reached when all the "stableoptimal" solutions for the highest 1-level $(l=1)$ are found.

\subsection{Calculation Process on the Sample of a Special Six-Level Hierarchical System}

All possible (in this six-level hierarchical system) $t$-full Scenarios should be considered. Each current $t^{\wedge}$ full Scenario represents a $t^{\wedge}$-combination of $l$-scenarios for all considered six levels of MVC series, where $l$-scenarios $(l=1, . ., 6)$ in this $t^{\wedge}$-combination are taken one at a time for each $l$-level $(l=1, \ldots, 6)$. These l-scenarios from $t^{\wedge}$-full Scenario are presented by a set of their numbers $\left\{k^{\wedge}, m^{\wedge}, n^{\wedge}, p^{\wedge}, r^{\wedge}, q^{\wedge}\right\}$.

The consideration of all $t$-full Scenarios or $t$-combinations $\{k, m, n, p, r, q\}$ in a framework of the calculation process should be performed as described below:

1. For a subset of $t$-full Scenarios, obtained by varying 6-scenarios in the framework of their totality $\{q=1, \ldots, \mathrm{Q}\}$ and by leaving unchanged all fixed scenarios $\left\{k^{\wedge}, m^{\wedge}, n^{\wedge}, p^{\wedge}, r^{\wedge}\right\}$ for all upper $l$-levels $(l=1, \ldots, 5)$, the appropriate set of subsets $\left\{S_{6}[q], q=1, \ldots, \mathrm{Q}\right\}$ will be determined. Each such subset $S_{6}\left[q^{\wedge}\right]$, corresponding to $t^{\wedge}$-full Scenario $\left\{k^{\wedge}, m^{\wedge}, n^{\wedge}, p^{\wedge}, r^{\wedge}, q^{\wedge}\right\}$, includes a predetermined number of sub-optimal alternatives, obtained by solving a mono-optimization problem, which is formed on a basis of a certain combination of criteria weight values, determined using Monte Carlo simulations.

2. The subset $S_{5}\left[r^{\wedge}\right]$ of "stable-optimal" alternatives is determined, using a special developed procedure (see section 4.2), on a basis of analysis of the derived set $\left\{S_{6}[q], q=1, . ., \mathrm{Q}\right\}$, corresponding to the combinations $\left\{k^{\wedge}, m^{\wedge}, n^{\wedge}, p^{\wedge}, r^{\wedge}, q / q=1, . ., \mathrm{Q}\right\}$ with fixed scenario numbers for five upper $l$-levels $(l=1, \ldots, 5)$. 3. By changing 5 -scenarios according to their numbers in the totality $\{r=1, \ldots, \mathrm{R}\}$, leaving unchanged all fixed scenarios $\left\{k^{\wedge}, m^{\wedge}, n^{\wedge}, p^{\wedge}\right\}$ for upper four $l$-levels $(l=1, . .4)$, we determine, by the above mentioned way, all possible subsets $\left\{S_{5}[r], r=1, \ldots, \mathrm{R}\right\}$, and, on their basis - the "stable-optimal" subset $S_{4}\left[p^{\wedge}\right]$.

4. Repeating the same operations for all 4-scenarios, we'll find the set of subsets $\left\{S_{4}[p], p=1, \ldots, \mathrm{P}\right\}$ for the fixed $\left\{k^{\wedge}, m^{\wedge}, n^{\wedge}\right\}$, and continuing further in this way, the set of subsets $\left\{S_{I}[k], k=1, \ldots, \mathrm{K}\right\}$, allowing to reach the required RAS as a "stable-optimal" subset of this set of subsets $\left\{S_{I}[k], k=1, \ldots, \mathrm{K}\right\}$. 


\subsection{The Procedure to Find "Stable" Solutions (Alternatives)}

Forming all $r$-variants $\left(r=1, . ., \mathrm{R}_{\mathrm{n}}\right)$ of each $n$-MVC series, corresponding to a considered $l$-level, is completed when a set of subsets $\left\{S_{r}, r=1, . ., \mathrm{R}_{\mathrm{n}}\right\}$ is obtained. Each its subset $S_{r}$ should include a predetermined number $N[r]$ of alternatives, chosen from the ISA using either a mono-optimization process (determining sub-optimal alternatives) or the procedure, considered below and allowing to find (on the basis of analysis of all subsets $\left\{S_{r}, r=1, \ldots, \mathrm{R}_{\mathrm{n}}\right\}$ ) a set (subset) $S^{\wedge}{ }_{n,}$, including a predetermined number $N^{\wedge}$ of "stable-optimal" alternatives (this set $S^{\wedge}{ }_{n}$ of "stable-optimal" solutions is determined independently for each considered $n$-MVC series).

These "stable-optimal" solutions from the set ${S^{\wedge}}_{n}$ should reflect the alternatives from the subsets $S_{r}$ $\left(r=1, . ., R_{n}\right)$, which have the highest frequency of appearing in the set of these subsets. So, if a full set $S^{\mathcal{M}_{n}}$, integrating all $g$-alternatives from the subsets $S_{r}\left(r=1, . ., R_{n}\right)$, has been constructed, then the frequency $P[g]$ of appearing in this full set $S^{\wedge}$ may be calculated for each considered $g$-alternative as:

$$
P[g]=n[g] / R_{n}, \quad g \in S^{\Lambda_{n}} ; \quad S^{\varkappa_{n}}=U\left\{S_{r}, r=1, . ., \mathrm{R}_{\mathrm{n}}\right\}
$$

where $n[g]$ is a number of subsets $\left\{S_{r}, r=1, . ., \mathrm{R}_{\mathrm{n}}\right\}$, including this $g$-alternative.

Thus, the following methodology is proposed to form the set $S_{n}^{\wedge}$ of "stable-optimal" alternatives:

1. The value $P[g]$, determined for each $g$-alternative from the set $S^{\wedge}{ }_{n}$ according to (7), is compared with the limit $P^{*}$, given a priori or by means of a dialogue (first, $P^{*}=1$ may be assigned).

2. If $P^{*}{ }_{n} \leq P[g]$, then the considered $j$-alternative is included into the set $S^{\wedge}$, otherwise we address to the next $g$-alternative from the set $S^{\wedge}$ in order to determine its value $P[g]$, and so on.

3. Once all $g$-alternatives from the set $S^{\wedge}{ }_{n}$ are checked, the number $N^{\wedge}{ }_{n}$ of $g$-alternatives, entering into the set $S_{n}^{\wedge}$, is compared with a limit $N^{*}$, given a priori or by dialogue for the considered $n$-MVC series.

4. If $N^{*} \leq N_{n}^{\wedge}$, the set $S_{n}^{\wedge}$ is already formed, otherwise either the limit $N^{*}{ }_{n}$ should be reduced or the uncompleted set $S_{n}^{\wedge}$ should be fixed.

\subsection{The Illustrative Example}

The above considered (section 4.1) calculation process will be illustrated on the two-criteria problem of selecting the "reasonable" solutions (the RAS) from the initial set of alternatives (the ISA), represented by 40 points each of which has two coordinates $\{x(g), y(g)\}$ (see Table 1). We will consider the minimization of such coordinate values as the optimization criteria. According to it and formula (5), we determine the following coordinates of the IS ("ideal" solution) and the WS ("worse" solution):

$$
Z^{\wedge}=\left\{z^{\wedge}=1.0, z^{\wedge}=1.0\right\} ; Z^{\wedge}=\left\{z^{\wedge}{ }_{I}=12.5, z^{\wedge}{ }_{2}=14.0\right\}
$$

Using these values and according to formula (6), we can determine the normalized criteria assessment vector for each alternative from the ISA, based on normalization of coordinate values. For example, such vector for the alternative 1 (point $x_{1}$ ) is $\{0 ; 0.96\}$, that is calculated as follows:

$$
\left[z_{1}\left(x_{1}\right)-z^{\wedge}{ }_{1},\right] /\left(z^{\wedge_{1}-z^{\wedge}}{ }_{1}\right)=[1.0-1.0] /(12.5-1.0)=0 ; \quad\left[z_{2}\left(x_{1}\right)-z^{\wedge}{ }_{2}\right] /\left(z^{\wedge}{ }_{2}-z^{\wedge}{ }_{2}\right)=[13.5-1.0] /(14.0-1.0)=0.96
$$

The following totalities of $l$-scenarios $(l=1-6)$ are in the considered six-level hierarchical system: a) For 1 -level $(l=1)$, there is only one 1 -scenario $1 *^{*} \cdot 1 \cdot k^{\wedge}\left(k^{\wedge}=1 ; \mathrm{K}=1\right)$, based on the successive performance of techniques TD.2 and TR.2 as well as one $k^{\wedge}$-version of the algorithm to construct the intervals of criteria weight values (see section 3.1).

b) For 2- and 3-level $(l=2,3)$, one version of the ISA $\left(m^{\wedge}=1 ; \mathrm{M}=1\right)$ and the same for the ISCAV $\left(n^{\wedge}=1\right.$; $\mathrm{N}=1$ ) are considered. Both these initial sets are represented by $40 \mathrm{~g}$-alternatives and their criteria values $x(g), y(g)$ (see in Table 1 the points $g=1-40$ and their coordinates, expressing the appropriate criteria values $x(g), y(g))$. All the normalized criteria assessment vectors from the ISCAV are calculated as indicated above for alternative (point) 1 (see formula (6.1)).

c) For 4-level $(l=4)$, three 4-scenarios represent three versions of "central points" of the intervals of criteria weight values $\left(p^{\wedge}=1,2,3 ; \mathrm{P}=3\right)$, assigned in a framework of used technique TD.2. These assigned versions of relative values are: 1) $\{0.5,0.5\} ; 2)\{0.65,0.35\} ; 3)\{0.35,0.65\}$ (section 3.3). 


\section{Table 1}

\section{The points and their coordinates reflecting the ISA and the ISCAV}

\begin{tabular}{|c|c|c|c|c|c|c|c|c|c|c|c|}
\hline \multicolumn{2}{|c|}{ |\# point| } & Values: & \multicolumn{2}{|c|}{ ||\# point| } & Values: & \multicolumn{2}{|c|}{ ||\# point| } & Values: & \multicolumn{3}{|c|}{ ||\# point| Values: | } \\
\hline$g$ & $x(g)$ & $y(g) \|$ & $g$ & $x(g)$ & $y(g)$ & $g$ & $x(g)$ & $y(g) \|$ & $g$ & $\mid x(g)$ & $y(g)$ \\
\hline 1 & 1.0 & & 1 & & 1 & 2 & 0.5 & & 3 & |10.0 & \\
\hline 2 & 2.25 & 12.5 & 12 & 5 & 1.0 & 22 & 8.75 & 5. & 32 & 7.25 & 10.0 \\
\hline 3 & 3.0 & 11 & 13 & & 1.5 & 23 & 10.75 & 5.5 & 3 & 9.75 & 10 \\
\hline 4 & 3.5 & 10.0 & 14 & 0 & 1.5 & 24 & 7.25 & $6.5 \|$ & 34 & 11.5 & 10.0 \\
\hline 5 & 4.0 & 9 & 15 & 5 & 2.5 & 25 & 9.25 & 6.5 & 35 & 8.75 & 10.5 \\
\hline 6 & 5.0 & 7.5 & 16 & 25 & 53.0 & 26 & 11.0 & 8.0 & 36 & 11.75 & 10.5 \\
\hline 7 & 5.5 & $6.5 \|$ & 17 & 9.75 & 53.0 & 27 & 5.75 & 8.0 & 37 & 6.5 & 12.0 \\
\hline 8 & 6.25 & $5.5 \|$ & 18 & 8.25 & 54.0 & 28 & 7.75 & 8.0 & 38 & 9.25 & 13.0 \\
\hline 9 & 6.5 & $4.0 \|$ & 19 & 9.75 & $\begin{array}{ll}5 & 4.0\end{array}$ & 29 & 10.0 & 8.0 & 39 & 10.75 & 13.0 \\
\hline 10 & 7.5 & $3.0 \|$ & 20 & 7.25 & $\begin{array}{ll}5 & 4.5\end{array}$ & | 30 & 11.5 & $8.0 \|$ & 40 & 8.75 & 14.0 \\
\hline
\end{tabular}

d) For 5-level $(l=5)$, three 5-scenarios represent three versions of bounds for the intervals of criteria weight values $\left(r^{\wedge}=1,2,3 ; \mathrm{R}=3\right)$, assigned in performance of the algorithm of constructing these bounds (see section 3.3). We use a "simple" version of such algorithm, consisting in a hypothetical assignment of the considered bounds (in relative values or \%) around the accepted (in 4-scenarios) "central points". Thus, the following bounds are considered: $(0.95,1.05) ;(0.9,1.1) ;(0.85,1.15)$.

e) For 6-level $(l=6)$, there is the number $G\left(G=K^{*} M^{*} N^{*} P^{*} R^{*} \mathrm{Q}\right.$ according to section 3$)$ of 6-scenarios, which should be equal to the number of all possible full Scenarios in this problem (see section 4). Each such full Scenario corresponds to a combination $\left\{k^{\wedge}, m^{\wedge}, n^{\wedge}, p^{\wedge}, r^{\wedge}, q^{\wedge}\right\}$ reflecting the numbers of $l$-scenarios for all $l$-levels $(l=1-6)$, taken one at a time for each $l$-level. Since we assign (a priori) $\mathrm{Q}=10$, the $20\left(\mathrm{Q}^{*} \mathrm{~J}\right.$, see section 3.3) Monte Carlo simulations are performed for each 6-scenario, and 180 $(\mathrm{G}=1 * 1 * 1 * 3 * 3 * 10 * 2=180)$ such simulations - for all possible full Scenarios.

On a basis of results derived using our developed computer model, we will illustrate the calculation process (see section 4.1), consisting in performance of all possible t-full Scenarios each of which is represented by the combination $\left\{k^{\wedge}, m^{\wedge}, n^{\wedge}, p^{\wedge}, r^{\wedge}, q^{\wedge}\right\}$. This illustration is as follows:

1. We begin to perform full Scenarios $\{1,1,1,1,1, q / q=1, . ., 10\}$, which are formed by choosing the following $l$-scenarios $(l=1-6)$ in the order "from top to bottom" (see sections 3, 4):

One) 1-scenario $1 . * 1 \cdot k^{\wedge}\left(k^{\wedge}=1\right)$, reflecting the unique $(\mathrm{K}=1)$ version of modified TOPSIS technique;

Two) 2- and 3-scenarios $\left(m^{\wedge}=1, n^{\wedge}=1\right)$, representing the unique $(\mathrm{M}=1, \mathrm{~N}=1)$ versions of the ISA and ISCAV, reflected by data from the Table 1;

Three) the first versions of 4- and 5-scenarios $\left(p^{\wedge}=1, r^{\wedge}=1\right)$, representing the same $j$-intervals $(j=1,2)$ of criteria weight values: $[0.95 * 0.5,1.05 * 0.5]=[0.425,0.525]$ around the same "central point" 0.5 ;

Four) $10(\mathrm{Q}=10)$ versions of 6-scenarios where each such $6\left(q^{\wedge}\right)$-scenario, corresponding to $q^{\wedge}$-series $\left(q^{\wedge}=1, . ., 10\right)$ of $\mathrm{J}(=2)$ Monte Carlo simulations, allows to form a subset $S_{6}\left[q^{\wedge}\right]$ of sub-optimal $g$ alternatives, using mono-optimization of the set of g-alternatives scalar estimates, which are in the left side of formula (6) and are not exceed the assigned limit $\mathrm{dR}=0.5$. Thus, varying all $q$-series of Monte Carlo simulations, we perform all full Scenarios $\{1,1,1,1,1, q / q=1, . ., 10\}$ and reach the whole set $\left\{S_{6}[q], q=1, \ldots, \mathrm{Q}\right\}$ of the appropriate "sub-optimal" subsets.

We will numerically illustrate these operations "Four" as follows: Using the following 1-series $\left(q^{\wedge}=1\right)$ of Monte Carlo simulations $(0.33514 ; 0.03326)$, we calculate the appropriate criteria weights: $W_{I}(1)=0.475+(0.525-0.475) * 0.33514=0.643 ; W_{2}(1)=0.475+0.5 * 0.03326=0.492$. Further, using them and criteria assessment vector (6.1), the scalar estimate of 1 -alternative $(g=1)$, represented in the left side of formula (6), is calculated: $0.643 * 0+0.492 * 0.96=0.472$. Such estimates for all $40 \mathrm{~g}$-alternatives are calculated by the same procedure, and after their comparison with the limit $d R=0.5$ (see (6)), the subset $S_{6}[1]$, including 12 alternatives $\{11,9,10,16,8,20,7,12,6,18,5,13\}$, is found using the above mentioned mono-optimization process (the number 12 of chosen alternatives is assigned a priori). Continuing these procedures, all of the subsets $\left\{S_{6}[1], . ., S_{6}[10]\right\}$ are determined. 
2. The subset $S_{5}[1]\left(r^{\wedge}=1\right)$, including 10 "stable-optimal" alternatives $\{11,9,10,16,20,8,7,12,18,6\}$, is determined on a basis of special analysis (section 4.2 with the assigned a priori $P_{n}^{*}=0.6, N^{*}=10$ ) of the obtained set $\left\{S_{6}[q], q=1, . ., 10\right\}$, corresponding to the full Scenarios $\{1,1,1,1,1, q / q=1, . ., 10\}$.

3 . Going to the next 5 -scenarios $\left(r^{\wedge}=2,3\right)$, connected with the new $j$-intervals $(j=1,2)$ of criteria weight values $[0.9 * 0.5,1.1 * 0.5]=[0.45,0.55],[0.425,0.575]$ around the same "central point" $0.5\left(p^{\wedge}=1\right)$, the new full Scenarios $\{1,1,1,1,2, q / q=1, . ., 10\},\{1,1,1,1,3, q / q=1, . .10\}$ are performed and the subsets $S_{5}[2]\left(r^{\wedge}=2\right)$, $S_{5}[3]\left(r^{\wedge}=3\right)$ are reached. Using the same special analysis for the set of these subsets $\left\{S_{5}[r], r=1,2,3\right\}$, the "stable-optimal" subset $S_{4}[1]\left(p^{\wedge}=1\right)$ is reached.

4. Continuing the above considered process for other 4 -scenarios $\left(p^{\wedge}=2,3\right)$, we'll find the subsets $S_{4}[2]$ $\left(p^{\wedge}=2\right), S_{4}[2] \quad\left(p^{\wedge}=3\right)$, corresponding to different "central points" $(0.65 ; 0.35) ;(0.35 ; 0.65)$ of the intervals of criteria weight values. This variation changes seriously the structure of the resulting "stable-optimal" subsets (e.g., $S_{4}[2]=\{1,2,3,4,5,9,6,7,8,10\}$ ). Since there is only one combination $\{1,1,1\}$ for external $l$ scenarios $(l=1,2,3\}$, the "stable-optimal" subset $S_{3}[1]$, including the set $\{9,11,10,8,20,7,16,12,5,6\}$, represents the required solution RAS. The final order of entering the alternatives into the RAS should reflect their quality, estimated with various positions. Accounting for this, 9-alternative is the best, 11alternative is the second in such classification, and so an.

\section{Preliminary Conclusions}

The approach is proposed to realize a multi-level hierarchical system of scenarios where each its level reflects its own kind of uncertainty and methods of accounting for it, that is adequate to the class of objects, considered on this level. The approach is suitable to solve various problems in different fields (economics, environment, etc.), having a considerable (maybe a vast) number of initial alternatives as well as an arbitrary number of criteria. The "bottleneck" in solving such real problems is connected with the construction of initial sets of alternatives (ISA) and criteria assessment vectors (ISCAV).

\section{References}

Kalika,V. and Frant, S. (1996) "Multicriteria analysis accounting for uncertainty factors in electricity generation expansion planning", Proceedings of 12th Power Systems Computation Conference, Dresden, Germany, 1,145-151.

Kalika,V., Frant,S.(1999) "Environmental Aspects of Power Generation", Energy Sources 21, 687-704.

Kalika,V.and Frant, S. (2000) "Multicriteria Optimization Accounting for Uncertainty in Dynamic Problem of Power Generation Expansion Planning", Research and Practice in Multiple Criteria Decision Making. (Eds. Haimes,Y.Y; Steuer, R.E.).In: LNEMS. Springer-Verlag, 487, 409-420.

Koksalan,M., Karvan,M.H., Zionts, S. (1988) "An Approach for Solving Discrete Alternative Multiple Criteria Problems involving Ordinal Criteria", Naval Research Logistics Quarterly, 35, 625-642.

Massam, B.N. (1988) "Multi-criteria decision making (MCDM): Techniques in planning", Progress in Planning, 30, 1-84.

Rajabi, S., Hipel, K.W., Kilgour D.M. (2000) "Multiple Criteria Subset Selection Under Quantitative and Non-Quantitative Criteria", Research and Practice in Multiple Criteria Decision Making (Eds: Haimes,Y.Y; Steuer, R.E.), In:LNEMS Springer-Verlag, 487, 137-148.

Steuer, R.E. (1986) .Multiple Criteria Optimization: Theory, Computation and Application, New York: Wiley. 